\title{
Los cambios de peso moderados provocan la modificación del número de apneas del sueño
}

Longitudinal Study of Moderate Weight Change and Sleep-Disordered Breathing. Peppard PE, Young T, Palta M. JAMA, 2000; 284(23) 3015-3021.

\section{Objetivo}

Valorar la asociación entre el cambio de peso y la modificación en la severidad de trastornos ventilatorios del sueño (TVS).

\section{Diseño}

Estudio de cohortes prospectivo y longitudinal con 4 años de seguimiento

\section{Lugar}

Wisconsin, Estados Unidos, entre Julio de 1989 y enero de 2000

\section{Participantes}

De 948 trabajadores residentes de Wisconsin que fueron seleccionados al azar, se realizó seguimiento efectivo durante 4 años, de 690 personas (edad media: 46 años, DS: 8 años, 385 hombres) que fue la población finalmente analizada. Los criterios de exclusión fueron embarazo, enfermedad grave cardiopulmonar, cáncer o cirugía reciente de vías aéreas.

\section{Evaluación de factores de riesgo}

Se realizó una valoración basal y una segunda evaluación a los cuatro años. Cada evaluación consistió en historia clínica, cuestionarios de estilo de vida, hábitos (tabaco, alcohol, etc.) y factores socio-demograficos, medidas antropométricas (peso, altura, índice masa corporal -IMC-, múltiples pliegues cutáneos, perímetros de cuello y cintura) y polisomnografia nocturna con oximetría (Índice de Apneas hipopneas -IAH = número de apneas e hipopneas por hora de sueño)

Medición de resultados principales

Comparando la evaluación basal con la realizada a los 4 años, se midió el cambio en el IAH (medido en porcentaje) y desarrollo de TVS moderado a severo (definido como un IAH > 15 apneas por hora de sueño) asociado al cambio en el peso.

\section{Resultados Principales}

En el modelo multivariable* de TVS ajustado por sexo, edad basal, IMC y hábito de fumar, el cambio de peso se relacionó positivamente con el cambio en el IAH. Para cambios moderados de peso (aumento o disminución) por cada $1 \%$ de cambio de peso se asoció con aproximadamente una media de $3 \%$ de cambio en el IAH. (ver Tabla) En valores extremos esta relación lineal se comportaba con un plateau.

El análisis no se modificó con el ajuste de otras variables (menopausia, ejercicio, alcohol, educación). Se asoció positivamente el IAH con cambios del IMC basal $(p<.001)$, perímetro de cuello $(p<.001)$ y cintura $(p<.001)$, y grosor total de pliegues $(p<.05)$.

\begin{tabular}{c|c|c}
\hline $\begin{array}{c}\text { Porcentaje del cambio } \\
\text { de peso (vs no cambio) }\end{array}$ & $\begin{array}{c}\text { Porcentaje Estimado en } \\
\text { cambio del IAH (95\% IC) }\end{array}$ & $\begin{array}{c}\text { Oddis Radio desarrollo de } \\
\text { TVS moderado a severo } \\
\text { (95\% IC) }\end{array}$ \\
\hline-20 & $-48(-58 \mathrm{a}-35)$ & - \\
\hline-10 & $-26(-34 \mathrm{a}-\mathbf{1 8})$ & - \\
\hline-5 & $-14(-18 \mathrm{a}-9)$ & - \\
\hline+5 & $+15(+10 \mathrm{a}+21)$ & $2.5(1.5-4.1)$ \\
\hline+10 & $+32(+20 \mathrm{a}+45)$ & $6.0(2.2-17.0)$ \\
\hline+20 & $+70(+42 \mathrm{a}+104)$ & $36.6(4.6->50)$ \\
\hline
\end{tabular}

\section{Conclusiones}

La disminución moderada de peso se asocia a disminución en el IAH y puede resultar efectiva para reducir el riesgo de TVS.

Fuente de financiamiento: INational Institutes of Health, EE.UU.

\section{Comentario}

Las apneas del sueño se asocian a un aumento significativo en la morbi/mortalidad cardiovascular y ventilatoria. La incidencia de apneas en adultos es de $1.5-5.7 \%$, con un pico de incidencia de $24 \%$ en mayores de 65 años $1-2$.

El presente trabajo tiene el mérito del tamaño de la población analizada y la amplia selección de variables clínicas consideradas con un seguimiento a 4 años. La utilización del método diagnóstico de referencia, la polisomnografía nocturna con oximetría (estudio de múltiples variables fisiológicas con alto consumo horas/hombre de operadores médicos y técnicos), impacta en los resultados obtenidos. El evitar la utilización de métodos simplificados (oximetría nocturna, estudios simplificados cardio-respiratorios, etc.) genera una diferencia categórica en relación a la sensibilidad y especificidad diagnóstica con una serie de otros trabajos publicados que usaron estos métodos. Teniendo en cuenta estas características del diseño, los resultados son concluyentes. Demuestra estadísticamente que la única de las variable evaluadas asociada a la gravedad del trastorno ventilatorio del sueño, es el peso corporal con una relación directamente propor- cional entre el peso y el IAH. Resulta interesante la cuantificación de esta relación lineal entre el porcentaje de modificación del peso con el porcentaje de modificación del IAH y que esta relación sea sólo para cambios moderados de peso (en $20 \%$ del peso en más o en menos). No se estratificó la gravedad del TVS para valorar el impacto de la modificación del peso en el seguimiento a 4 años.

Dentro del contexto de los TVS debemos tener presente que la disminución de peso como tratamiento único es ineficaz para normalizar el IAH, es decir, lograr la supresión del cuadro. Esto sólo puede lograrse con el uso de CPAP (continuous positive airway pressure) nasal nocturno. Sin embargo la disminución de peso es un factor que siempre debe corregirse y puede ser el único tratamiento para cuadros iniciales (roncopatía primaria o síndrome de resistencia primaria de vía aérea superior) en situaciones particulares. Debemos considerar al exceso de peso como un factor más ya que en la producción de apneas participan un número de eventos fisiológicos que no pueden ser evaluados clínicamente en estudios epidemiológicos.

\section{Dr. Guillermo L. Zanniello}

[ Jefe de Sección: Unidad Alteraciones del Sueño y Centro de Epilepsia Servicio de Neurología del Hospital Italiano de Buenos Aires ]

Referencias

1. Sleep apnea. Bradley TD., Floras JS. New York 2000

2. Sleep Medicine: Principles and Practice. Kryger, Roth, Dement. Third Edition. Philadelphia. 2000 Sleep Medicine. Aldrich M. Oxford University Press. 1999 